\title{
An inverse problem for the estimation of upstream velocity profiles in an incompressible turbulent boundary layer
}

\author{
Wagner M. Brasil ${ }^{\text {a }}$, Jian $\mathrm{Su}^{\mathrm{b}, *}$, Atila P. Silva Freire ${ }^{\mathrm{c}}$ \\ a Centro Tecnológico do Exército, Av. das Américas 28705, Guaratiba, 23020-470 Rio de Janeiro, Brazil \\ ${ }^{\mathrm{b}}$ Nuclear Engineering Program, COPPE, Universidade Federal do Rio de Janeiro, C.P. 68509, 21945-970 Rio de Janeiro, Brazil \\ ${ }^{\mathrm{c}}$ Mechanical Engineering Program, COPPE, Universidade Federal do Rio de Janeiro, C.P. 68503, 21945-970 Rio de Janeiro, Brazil
}

Received 29 May 2003; received in revised form 6 September 2003

\begin{abstract}
An inverse problem is solved for the estimation of upstream velocity profiles in an incompressible turbulent boundary layer over a smooth flat plate. The inverse analysis is based on the boundary layer morphology, making use of the law of the wall and the law of the wake to estimate boundary layer parameters from measured velocity histories. The direct problem of the turbulent boundary layer equations is solved by using finite difference method with the Cebeci-Smith turbulence model. The numerical solution of the direct problem is validated by experimental data obtained through the hotwire anemometry in a low-speed wind tunnel. The friction velocity, Von Kármán constant, law of the wall constant, Coles's wake-strength parameter and boundary layer thickness for the initial profile are determined as unknown parameters by the Levenberg-Marquardt algorithm. The estimated upstream velocity profiles compare favourably with hotwire anemometry measurements at the same location.

(c) 2003 Elsevier Ltd. All rights reserved.
\end{abstract}

Keywords: Turbulent boundary layer; Inverse problem; Hotwire anemometry; Parameter estimation; Levenberg-Marquardt algorithm

\section{Introduction}

Inverse problems have originated in the heat transfer community in connection with the estimation of surface heat flux histories from measured temperature histories inside a heat conducting body. Inverse heat conduction problems have been studied extensively for estimation of unknown boundary or initial conditions, thermophysical properties, heat source strength, and geometrical configuration [1-6]. A variety of numerical and analytical techniques have been developed for the solution of inverse heat conduction problems, for example, the function specification method, the Tikhonov regularisation method, the mollification method, and the Alifanov's iterative regularisation method.

\footnotetext{
* Corresponding author. Tel.: +55-21-2562-8448; fax: +5521-2562-8444/8450.

E-mail address: sujian@con.ufrj.br (J. Su).
}

Despite many potential applications, inverse heat convection problems have only recently received some attention. In convective environments, early studies were carried out by Cebeci and coworkers [7-9] in connection with the determination of the spatial variation of the flow freestream velocity for a given local wall shear stress. However, as recognised by Moutsouglou [10], Cebeci and his coworkers failed to capture the ill-posed nature of the problem as the calculated values of the direct problem were used as boundary conditions for the inverse problem. This procedure caused an unnecessary contamination of the inverse problem that made its results difficult to assess. Moutsouglou [10] apparently was the first to address an inverse convection problem, using a sequential function specification algorithm for the estimation of the asymmetric heat flux in steady state mixed convection in a vertical channel. The same author has also applied the whole domain regularisation technique in an inverse analysis to estimate wall heat flux in an elliptic laminar forced convection problem [11]. 


\begin{tabular}{|c|c|c|c|}
\hline \multicolumn{4}{|c|}{ Nomenclature } \\
\hline$A$ & law of the wall constant & $y^{+}$ & dimensionless spatial coordinate normal to \\
\hline$c_{\mathrm{f}}$ & skin-friction coefficient & & the plate $\left(=y u_{\tau} / v\right)$ \\
\hline$D$ & diagonal matrix & $Z_{m}$ & measured velocity \\
\hline$F_{m}$ & difference between calculated and measured & & \\
\hline & velocities & \multicolumn{2}{|c|}{ Greek symbols } \\
\hline$J$ & Jacobian matrix & $\delta$ & boundary layer thickness \\
\hline$M$ & number of velocity measurement points & $\delta_{2}$ & momentum boundary layer thickness \\
\hline$R$ & squared residue & $\kappa$ & Von Kármán constant \\
\hline$R_{\delta 2}$ & Reynolds number based on the momentum & $\lambda$ & damping factor \\
\hline & boundary layer thickness & $\epsilon$ & convergence criterion \\
\hline$u$ & velocity component parallel to the plate & $v$ & kinematic viscosity of fluid \\
\hline$u_{e}$ & freestream velocity & $\Pi$ & Cole's wake-strength \\
\hline$u_{m}$ & calculated velocity at a measurement point & & \\
\hline$u_{\tau}$ & friction velocity & \multicolumn{2}{|c|}{ Subscripts } \\
\hline$v$ & velocity component normal to the plate & 0 & inlet \\
\hline$x$ & spatial coordinate along the plate & $k$ & iteration index \\
\hline$y$ & spatial coordinate normal to the plate & $n$ & index of unknown parameter \\
\hline
\end{tabular}

Inverse problems of laminar forced convection in ducts have been studied for estimation of initial temperature profile, wall heat flux and thermophysical properties [12-28]. On the other hand, few works have been published on inverse problems in turbulent flows despite its obvious technological relevance. Liu and Özişik [29] applied the conjugate gradient method with an adjoint equation for solving an inverse turbulent convection problem of estimating the timewise varying wall heat flux in parallel plate ducts. Recently, Li and Yan [30] solved an inverse problem for estimation of space- and time-dependent heat flux in turbulent forced convection between parallel flat plates, with the conjugate gradient method. Su et al. [31] applied the Levenberg-Marquardt method to estimate the nonuniform wall heat flux in a steady state, thermally developing, hydrodynamically developed turbulent flow in a circular pipe based on temperature measurements obtained at several different locations in the stream. Later, Su and Silva Neto [32] solved an inverse heat convection problem to estimate simultaneously the inlet temperature profile and the wall heat flux distribution in a steady state, thermally developing, hydrodynamically developed turbulent flow in a circular pipe based on temperature measurements obtained at several different positions in the stream, using the Levenberg-Marquardt method [33-35].

The purpose of the present work is to solve an inverse problem for the estimation of upstream velocity profiles for an incompressible turbulent boundary layer over a smooth flat plate. The solution procedure aims at developing a method which can be used confidently to predict local and global parameters of the flow. As recorded by Cebeci [9], "a slight error in the experimental skin-friction coefficient will severely affect the computed velocity distribution". Of course, the same remark is valid if we consider the computed skin-friction coefficient. In fact, the solution sensitivity on the chosen value of the skin-friction is known to be high for turbulent flows and a classical way to overcome this difficulty is to appeal to the asymptotic two-deck structure of the turbulent boundary layer. Here, the unknown upstream velocity profile will be represented by the composite Coles's law of the wall, law of the wake profile; then, the friction velocity, Von Kármán's constant, the law of the wall constant, Coles's wake-strength parameter and the boundary layer thickness for the initial profile, which will be determined as unknown parameters by using the Levenberg-Marquardt algorithm. The solution procedure will resort to velocity measurements obtained at several different downstream locations in the stream; the measurements were obtained through the hotwire anemometry technique. The effects on solution concerning the location of the measurement station are examined. The direct problem, presented in the next section, is solved by a finite difference method that uses the CebeciSmith turbulence model.

\section{Mathematical formulation of the direct problem}

The Reynolds averaged equations for a steady, incompressible and two-dimensional turbulent boundary layer can be written as follows: 
$\frac{\partial u}{\partial x}+\frac{\partial v}{\partial y}=0$

$u \frac{\partial u}{\partial x}+v \frac{\partial u}{\partial y}=-\frac{1}{\rho} \frac{\mathrm{d} p}{\mathrm{~d} x}+\frac{\partial}{\partial y}\left(v \frac{\partial u}{\partial y}-\overline{u^{\prime} v^{\prime}}\right)$.

The notation is classical. The algebraic turbulence model of Cebeci-Smith is used for the closure of the Reynolds stress (see [36]).

The partial differential equations are to be solved with the appropriate boundary conditions,

$u=0$ and $v=0$ for $y=0$,

$u=u_{e}(x)$ as $y \rightarrow \infty$,

$u=u_{0}(y)$ and $v=v_{0}(y)$ for $x=x_{0}$.

If the fluid properties, coefficients of turbulent modelling, and boundary conditions are known, the direct problem given by Eqs. (1)-(5) can be solved to obtain the velocity field of the turbulent boundary layer. In this work, the direct problem defined by Eqs. (1)-(5) is solved through a second-order implicit finite difference method [37].

\section{Solution of the inverse problem}

In the inverse problem considered in this work, we are looking for an unknown upstream velocity profile $u_{0}(y)$; this must be evaluated from velocity measurements taken at several downstream points in the flow field.

The unknown upstream velocity profile is represented by the composite Coles's law of the wall, law of the wake formulation [38]

$u_{0}(y)=u_{\tau}\left[\frac{1}{\kappa} \ln y^{+}+A+\frac{2 \Pi}{\kappa} \sin ^{2}\left(\frac{\pi}{2} \frac{y}{\delta}\right)\right]$,

where $\kappa$ (Von Kármán constant), $A$ (law of the wall constant), $u_{\tau}$ (friction velocity), $\Pi$ (Cole's wakestrength) and $\delta$ (boundary layer thickness) are parameters to be determined, and $y^{+}=y u_{\tau} / v$.

Upon the parameterisation given by Eq. (6), the inverse problem has been formulated as a parameter estimation problem. The solution of this inverse problem for the estimation of the five unknown parameters is based on the minimisation of the ordinary least squares norm defined by

$R(\vec{P})=\sum_{m=1}^{M}\left[u_{m}(\vec{P})-Z_{m}\right]^{2}$,

where $u_{m}(\vec{P})$ are the calculated velocities and $Z_{m}$ are the measured velocities at points $\left(x_{m}, y_{m}\right), m=1,2, \ldots, M$, with $M$ being the total number of measurement points.
The vector of unknown parameters is formed by

$\vec{P}^{\mathrm{T}}=\left[p_{1}, p_{2}, p_{3}, p_{4}, p_{5}\right]=\left[\kappa, A, u_{\tau}, \Pi, \delta\right]$.

We use the Levenberg-Marquardt method [33-35] for parameter estimation, written in matrix form

$\left(J^{\mathrm{T}} J+\lambda D\right) \Delta \vec{P}=-J^{\mathrm{T}} \vec{F}$,

where $D$ represents the diagonal matrix, $\lambda$ is a damping factor to improve the convergence behaviour and the elements of the Jacobian matrix are

$J_{m n}=\frac{\partial u_{m}}{\partial p_{n}}, \quad m=1,2, \ldots, M$ and $n=1, \ldots, 5$.

Eq. (9) is then written in a form convenient to be used in an iterative procedure,

$\Delta P^{k}=-\left(J^{k \mathrm{~T}} J^{k}+\lambda^{k} D^{k}\right)^{-1} J^{k \mathrm{~T}} \vec{F}^{k}$,

where $k$ is the iteration index.

A new estimation of the parameters, $\vec{P}^{k+1}$, is calculated by

$\vec{P}^{k+1}=\vec{P}^{k}+\Delta \vec{P}^{k}$

The iterative procedure starts with an initial guess for parameters, $\vec{P}^{0}$, and new estimates, $\vec{P}^{k+1}$ are sequentially obtained using Eq. (12) with $\Delta \vec{P}^{k}$ given by Eq. (11) until the convergence criterion

$\left|\frac{\Delta p_{n}^{k}}{p_{n}^{k}}\right|<\epsilon, \quad n=1, \ldots, 5$

is satisfied, where $\epsilon$ is a small real number, such as $10^{-8}$. The elements of the Jacobian matrix as well as the right hand term of Eq. (9) are calculated by using the solution of the direct problem defined by Eqs. (1)-(5), as described in the previous section.

\section{The classical approach}

For the simple case of a turbulent flow over a flat plate at zero incidence, approximate methods based on the momentum integral equation can be easily derived for the estimation of some flow parameters. In these methods, the boundary layer thickness is approximated by a suitable empirical equation; then, if the velocity distribution is considered to follow a certain form, the momentum equation can be integrated to provide a relation between the displacement thickness, momentum thickness and shear stress at the wall.

The assumption of a $1 / 7$ th power law of velocity distribution advanced by Prandtl relied on the idea that small differences in the velocity profile are not important since the drag will be evaluated from an integral. Thus, he considered that the velocity distribution in the boundary layer on a plate is identical with that inside a circular pipe. 
Hence, integration of the momentum equation from the initial value $\delta=0$ at $x=0$ furnishes (see [39])

$\delta_{2}=0.036 x\left(\frac{U_{\infty} x}{v}\right)^{-1 / 5}$,

$\delta=\frac{72}{7} \delta_{2}$

$c_{\mathrm{f}}=0.0576 \frac{u_{e} x}{v}$,

$u_{\tau}=u_{e} \sqrt{\frac{c_{\mathrm{f}}}{2}}$.

The above four equations together with the composite law of the wall/law of the wake can now be used to evaluate the velocity profile at any location from a given velocity profile at any other location. The steps are the following:

- From a given experimental velocity profile, calculate $\delta_{2}$.

- From Eq. (14), calculate the distance of the experimental velocity profile to a virtual plate origin.

- From Eq. (14), calculate $\delta_{2}$ for the unknown profile.

- From Eq. (15), calculate $\delta$ for the unknown profile.

- From Eqs. (16) and (17), calculate $u_{\tau}$ for the unknown profile.

- From Eq. (6), construct the unknown velocity profile.

To implement Eq. (6) in the classical approach one needs to know the values of parameters $\kappa, A$ and $\Pi$. Here, the following values were considered:

$\kappa=0.4$,

$A=5.0$,

$\Pi=-0.05757 \ln ^{2} R_{\delta 2}+1.062 \ln R_{\delta 2}-4.317$,

$R_{\delta 2}<5600$,

$\Pi=0.55, \quad R_{\delta 2} \geqslant 5600$.

\section{Experimental apparatus and instrumentation}

The experiments were carried out in a low-speed wind tunnel located at the Laboratory of Turbulence Mechanics of COPPE/UFRJ. The wind tunnel is of open circuit type and has a $5 \mathrm{~m}$ long test section with square cross section of $0.67 \mathrm{~m} \times 0.67 \mathrm{~m}$. Wind speed is continuously variable from 0.5 to $3.5 \mathrm{~m} / \mathrm{s}$. The turbulent intensity level in the freestream was about $1.0 \%$. Mean velocity profiles and turbulent intensity levels were measured by using a DANTEC series 55M hotwire anemometer with a standard P15 probe. A Pitot tube, a high precision inclined multi-tube manometer, and a computer controlled traverse gear were also used. Out- put signals of the hotwire anemometer were transmitted to a PC through a 16-bit data acquisition card.

An uncertainty analysis of the data was performed according to the procedure described in [40]. The uncertainty associated with the velocity measurements was: $U=0.064 \mathrm{~m} / \mathrm{s}$ precision, 0 bias $(P=0.95)$.

Six longitudinal velocity profiles were measured at stations $3.20,3.25,3.30,3.35,3.40$ and $3.45 \mathrm{~m}$ from the beginning of the test section. All profiles were measured over the central line of the test section. Around 60 mean velocity measurement points were taken for each profile.

The friction velocity $\left(u_{\tau}\right)$, Coles's wake-strength parameter $(\Pi)$, boundary layer thickness $(\delta)$, Von Kármán constant $(\kappa)$ and the law of the wall constant $(A)$ for each measured velocity profile were obtained through a program specially developed in the Mathematica ${ }^{\mathrm{TM}}$ software package environment.

\section{Results}

The study was developed in three parts. The objective of the first part was to validate the numerical solution for the direct problem by comparison with some experimental data. The velocity profile measured at station $3.20 \mathrm{~m}$ was used as the initial condition for the calculation of velocity profiles at the same stations where the measurements were performed. Fig. 1 shows a comparison between velocity profiles obtained through the numerical simulation and the experimental profiles at station $3.45 \mathrm{~m}$.

The second part aimed at estimating the upstream velocity profile, at station $x=3.20 \mathrm{~m}$, by the inverse method. We have carried out an analysis of the sensitivity coefficients, before the estimation of the unknown parameters. As the parameters involved in the inverse problem have different orders of magnitude and dimension $(k, A$ and $\Pi$ are dimensionless, $\delta$ has unit of

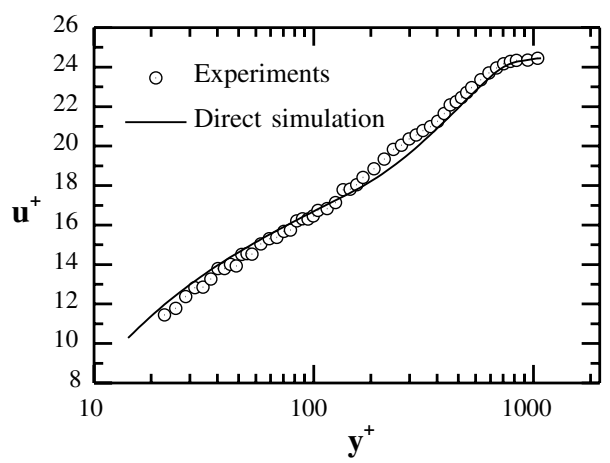

Fig. 1. Validation of the numerical solution for the direct problem. Comparison of calculated and measured velocity profiles in inner variable. 
length and $u_{\tau}$ unit of velocity), we chose to represent the results with relative sensitivity coefficients, defined as

$J_{p_{n}}=p_{n} \frac{\partial u}{\partial p_{n}}$.

Fig. 2 shows the results of the relative sensitivity coefficients, determined by using finite difference approximation with central difference. The sensitivity coefficients of the parameters $\delta$ and $A$ presented small magnitude. The dashed line represents the ratio between the sensitivity coefficient of the parameter $u_{\tau}$ and the sensitivity coefficient of the parameter $\kappa$. We can observe that this ratio is close to the value -1 along all the points, which shows that the sensitivity coefficients of these two parameters presented nearly symmetrical distributions and are consequently linearly dependent.

The analysis of the sensitivity coefficients described above shows that only the parameters $u_{\tau}$ and $\Pi$ could be estimated together. To overcome this difficulty, we developed a sequential method of parameter estimation by which the five parameters were progressively estimated. In this way, the unknown parameters $u_{\tau}$ and $\Pi$ were estimated first with the parameter $\delta$ being the value of the downstream experimental profile and the parameters $\kappa$ and $A$ the classical values described respectively in Eqs. (18) and (19). The parameter $\kappa$ was estimated in the next, with the values of the parameters $u_{\tau}$ and $\Pi$ already estimated, with the parameter $\delta$ being the value of the downstream experimental profile and the parameter $A$ the classical value. The parameter $\delta$ was then estimated with the values of the parameters $u_{\tau}, \Pi$ and $\kappa$ already estimated, with the parameter $A$ being the classical value. Finally, the parameter $A$ was estimated with the values of the parameters $u_{\tau}, \Pi, \kappa$ and $\delta$ already estimated.

A single experimental profile at a downstream station was used in the inverse analysis. We successfully estimated the upstream velocity profile using measured

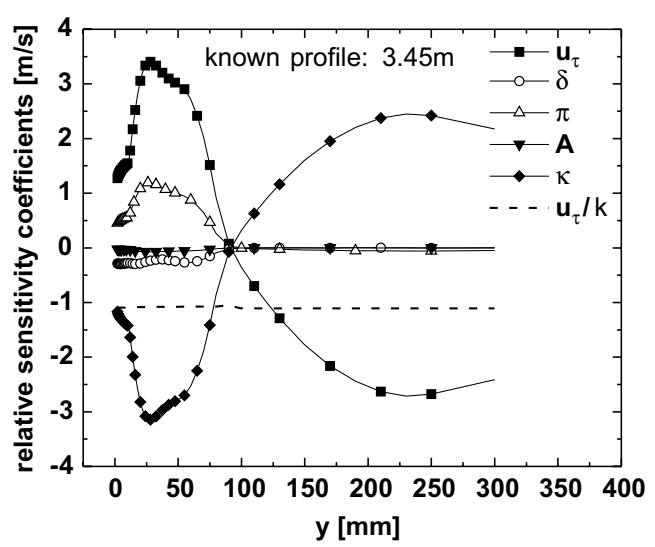

Fig. 2. Relative sensitivity coefficients.

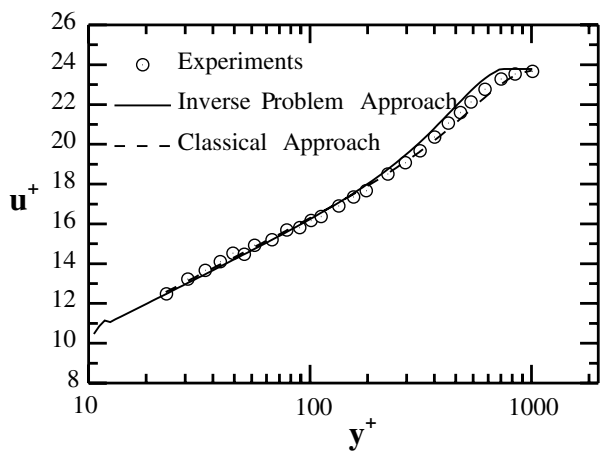

Fig. 3. Comparison of estimated initial velocity profile in inner variable with experimental data using one measured at station $3.45 \mathrm{~m}$.

profiles at stations $x=3.25,3.35$ and $3.45 \mathrm{~m}$. These profiles were then to be compared with the profile obtained experimentally and by the classical approach. Fig. 3 shows that the estimated upstream velocity profile agrees quite well with the measured upstream profile.

The $99 \%$ confidence interval for the estimated parameters was obtained from the diagonal elements of the covariance matrix, $\left[J^{\mathrm{T}} J\right]_{n n}^{-1}$, as follows (see [6]):

$$
\begin{aligned}
p_{n} & -2.576 \sigma \sqrt{\left[J^{\mathrm{T}} J\right]_{n n}^{-1}} \leqslant P_{n} \leqslant p_{n} \\
& +2.576 \sigma \sqrt{\left[J^{\mathrm{T}} J\right]_{n n}^{-1}}, \quad n=1, \ldots, 5,
\end{aligned}
$$

where $P$ is the real value of the parameter and $\sigma$ is the standard deviation of the measurement errors.

Table 1 presents the $99 \%$ confidence intervals for some values of standard deviation of the measurement errors, where $u_{e}=3.64 \mathrm{~m} / \mathrm{s}$ is the freestream velocity measured downstream at $x=3.45 \mathrm{~m}$. As the parameter $u_{\tau}$ presents a smaller diagonal element of the covariance matrix, $\left[J^{\mathrm{T}} J\right]_{n n}^{-1}$, than the other parameters, it is consequently less influenced by the measurement errors; on the contrary, more accurate measurements are necessary for the estimation of the parameters $\Pi$ and $A$, due to their larger diagonal element of the covariance matrix, $\left[J^{\mathrm{T}} J\right]_{n n}^{-1}$.

In the third part, we checked the precision of the numerical simulation of the turbulent boundary layer, as a direct problem, if the estimated initial profile was used as the initial condition. We used the estimated values of parameters $u_{\tau}, \kappa, A, \Pi$ and $\delta$ to construct the initial condition and compared the results with that obtained by using the directly measured initial profile. Fig. 4 shows a comparison between the velocity profiles at station $3.45 \mathrm{~m}$ when: (i) an inverse initial profile is used as an initial condition, (ii) the classical approach is used to find the initial condition.

The friction velocity is a flow parameter that is notoriously difficult to determine experimentally. In this 
Table 1

Confidence intervals of parameter estimation

\begin{tabular}{lllll}
\hline Parameter & Value estimated & $\sqrt{\left[J^{\mathrm{T}} J\right]_{n n}^{-1}}$ & $\sigma$ & $99 \%$ confidence interval \\
\hline$u_{\tau}$ & 0.153 & 0.013720 & $0.01 u_{e}$ & $0.1517 \leqslant u_{\tau} \leqslant 0.1543$ \\
& & $0.03 u_{e}$ & $0.1491 \leqslant u_{\tau} \leqslant 0.1569$ \\
& & $0.05 u_{e}$ & $0.1466 \leqslant u_{\tau} \leqslant 0.1594$ \\
$\delta$ & 0.0637 & 0.044868 & $0.01 u_{e}$ & $0.05949 \leqslant \delta \leqslant 0.06791$ \\
& & & $0.03 u_{e}$ & $0.05106 \leqslant \delta \leqslant 0.07634$ \\
& & & $0.05 u_{e}$ & $0.04264 \leqslant \delta \leqslant 0.08476$ \\
$A$ & 4.964 & & $0.01 u_{e}$ & $4.8514 \leqslant A \leqslant 5.0766$ \\
& & & $0.03 u_{e}$ & $4.6263 \leqslant A \leqslant 5.3018$ \\
$\kappa$ & 0.414 & $0.05 u_{e}$ & $4.4012 \leqslant A \leqslant 5.5268$ \\
& & & $0.01 u_{e}$ & $0.4107 \leqslant \kappa \leqslant 0.4173$ \\
& & & $0.03 u_{e}$ & $0.4042 \leqslant \kappa \leqslant 0.4238$ \\
$\Pi$ & 0.03478 & $0.05 u_{e}$ & $0.3977 \leqslant \kappa \leqslant 0.4303$ \\
& & & $0.01 u_{e}$ & $0.5043 \leqslant \Pi \leqslant 0.6957$ \\
& & & $0.03 u_{e}$ & $0.3129 \leqslant \Pi \leqslant 0.8871$ \\
& & $0.05 u_{e}$ & $0.1215 \leqslant \Pi \leqslant 1.0785$ \\
\hline
\end{tabular}

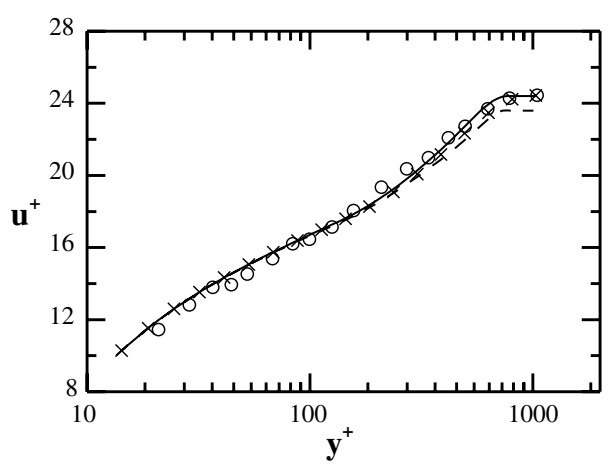

Fig. 4. Calculated downstream velocity profiles using experimental and estimated initial profiles; inner variables. Circles denote experiments; line, initial profile given by inverse method; dashed line, initial profile given by classical approach; $\times$, initial profile given by experiments.

work, the friction velocity was determined by means of a nonlinear regression program developed in the Mathematica software package for treatment of the experimental data.

Table 2 , in addition, shows values of friction velocity estimated by the inverse method compared with the measured values of friction velocity at station $x=3.20$ $\mathrm{m}$. As can be seen, the relative errors for $u_{\tau}$ were less than $5 \%$. This is a clear indication that inverse analysis can be used successfully to determine the friction velocity from mean velocity measurements in the downstream flow field.

Figs. 5 and 6 show the predicted and measured values of $c_{\mathrm{f}}$ and of $\delta_{2}$. The inverse method shows a clear ad-
Table 2

Comparison of flow parameters at $x=3.20 \mathrm{~m}$

\begin{tabular}{llll}
\hline Parameter & Experiments & $\begin{array}{l}\text { Inverse problem } \\
(x=3.45 \mathrm{~m})\end{array}$ & $\begin{array}{l}\text { Classical } \\
\text { approach }\end{array}$ \\
\hline$u_{\tau}(\mathrm{m} / \mathrm{s})$ & 0.160 & 0.153 & 0.162 \\
$\delta(\mathrm{m})$ & 0.0728 & 0.0637 & 0.0753 \\
$A$ & 5.247 & 4.964 & 5.00 \\
$\kappa$ & 0.420 & 0.414 & 0.410 \\
$\Pi$ & 0.495 & 0.600 & 0.445 \\
\hline
\end{tabular}

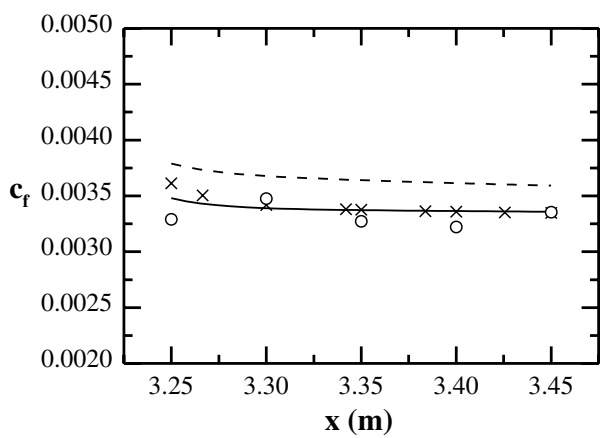

Fig. 5. Estimation of friction coefficient using one measured station, $3.45 \mathrm{~m}$. Circles denote experiments; line, inverse method; dashed line, classical approach; $\times$, direct method.

vantage over the classical approach. In fact, for the present conditions, the classical approach tends to underestimate the values of $\delta$, and that results in higher predicted values of $c_{\mathrm{f}}$. 


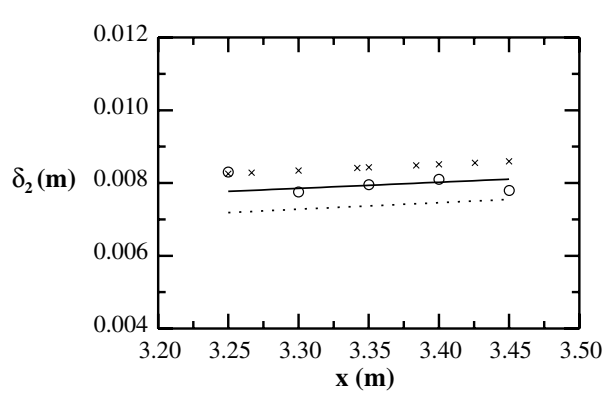

Fig. 6. Estimation of momentum thickness using one measured station, $3.45 \mathrm{~m}$. Circles denote experiments; line, inverse method; dashed line, classical approach; $\times$, direct method.

\section{Conclusion}

An inverse analysis for the estimation of upstream velocity profiles in an incompressible turbulent boundary layer over a smooth flat plate was carried out. The turbulent boundary layer direct problem with an algebraic turbulence model was solved through a finite difference method, which was validated against data obtained in a low-speed wind tunnel. The inverse problem for the estimation of initial velocity profiles was formulated as a parameter estimation problem that searched for the friction velocity, the Von Kármán constant, the law of the wall constant, the Coles's wakestrength parameter and the boundary layer thickness at an upstream station in the turbulent boundary layer. We have shown, through comparison with the measured velocity profile at the same station, that the upstream velocity profile can be accurately estimated if experimental data of velocity measurement within $25 \mathrm{~cm}$ from the inlet station are used. The proposed inverse analysis can therefore be used to generate an accurate and smooth initial velocity profile for numerical simulation of turbulent boundary layer and to determine accurately some boundary layer parameters, such as the friction velocity and the boundary layer momentum thickness, that are difficult to measure directly.

All the above results are very promising, which leads us to believe that an extension of the present procedure to the problem of turbulent boundary layers over rough surfaces may be possible. In that case, a new parameter, the error in origin, $\varepsilon$, will have to be considered in our analysis. Due to the great difficulties in experimentally assessing $\varepsilon$ (see, e.g. [41]), inverse methods may, therefore, become a powerful tool for the description of flows over rough surfaces. This issue will be addressed in the future by the present authors.

Finally, it should be pointed out that the present procedure can be easily extended so that the estimation of upstream temperature profiles can be made from measured temperature profiles.

\section{Acknowledgements}

The authors acknowledge the financial support provided by the Brazilian National Research Council (CNPq) and the Brazilian Ministry of Education (MEC/ CAPES).

\section{References}

[1] A.N. Tikhonov, V.Y. Arsenin, Solutions of Ill-Posed Problems, Winston \& Sons, Washington, DC, 1977.

[2] J.V. Beck, B. Blackwell, C.R. St. Clair, Inverse Heat Conduction: Ill-Posed Problem, Wiley, New York, 1985.

[3] E. Hensel, Inverse Theory and Application to Engineers, Prentice-Hall, Englewood Cliffs, NJ, 1991.

[4] D.A. Murio, The Mollification Method and the Numerical Solution of Ill-Posed Problems, Wiley, New York, 1993.

[5] O.M. Alifanov, Inverse Heat Transfer Problems, SpringerVerlag, New York, 1994.

[6] M.N. Özişik, H.R.B. Orlande, Inverse Heat Transfer: Fundamentals and Applications, Taylor \& Francis, New York, 2000.

[7] H.B. Keller, T. Cebeci, An inverse problem in boundarylayer flows: numerical determination of pressure gradient for a given wall shear, J. Comput. Phys. 10 (1972) 151-161.

[8] T. Cebeci, N. Berkant, I. Silivri, H.B. Keller, Turbulent boundary layers with assigned wall shear, Comput. Fluids 3 (1975) 37-49.

[9] T. Cebeci, An inverse boundary layer method for compressible laminar and turbulent boundary layers, J. Aircr. 13 (1976) 709-717.

[10] A. Moutsouglou, An inverse convection problem, J. Heat Transfer 111 (1989) 37-43.

[11] A. Moutsouglou, Solution of an elliptic inverse convection problem using a whole domain regularization technique, AIAA J. Thermophys. 4 (1990) 341-349.

[12] R. Raghunath, Determining entrance conditions from downstream measurements, Int. Commun. Heat Mass Transfer 20 (1993) 173-183.

[13] J.C. Bokar, M.N. Özişik, Inverse analysis for estimating the time varying inlet temperature in laminar flow inside a parallel plate duct, Int. J. Heat Mass Transfer 38 (1995) $39-45$.

[14] I. Gejadze, Y. Jarny, An inverse heat transfer problem for restoring the temperature field in a polymer melt flow through a narrow channel, Int. J. Therm. Sci. 41 (2002) $528-535$.

[15] C.H. Huang, M.N. Özişik, Inverse problem of determining unknown wall heat flux in laminar flow through a parallel plate, Numer. Heat Transfer, Part A 21 (1992) 55-70.

[16] H.A. Machado, H.R.B. Orlande, Inverse analysis of estimating the timewise and spacewise variation of the wall heat flux in a parallel plate channel, Int. J. Numer. Meth. Heat Fluid Flow 7 (1997) 696-710.

[17] H.-Y. Li, W.-M. Yan, Estimation of wall heat flux in an inverse convection problem, J. Thermophys. Heat Transfer 13 (1999) 394-396.

[18] H.A. Machado, H.R.B. Orlande, Inverse problem for estimating the heat flux to a non-Newtonian fluid in a 
parallel plate channel, J. Brazil. Soc. Mech. Sci. 20 (1998) 51-61.

[19] H.M. Park, J.H. Lee, A method of solving inverse convection problems by means of mode reduction, Chem. Eng. Sci. 53 (1998) 1731-1744.

[20] C.H. Huang, W.C. Chen, A three-dimensional inverse forced convection problem in estimating surface heat flux by conjugate gradient method, Int. J. Heat Mass Transfer 43 (2000) 3171-3181.

[21] H.-Y. Li, W.-M. Yan, Inverse convection problem for determining wall heat flux in annular duct flow, J. Heat Transfer 122 (2000) 460-464.

[22] M.J. Colaço, H.R.B. Orlande, Inverse forced convection problem of simultaneous estimation of two boundary heat fluxes in irregularly shaped channels, Numer. Heat Transfer, Part A 39 (2001) 737-760.

[23] P.-T. Hsu, C.-K. Chen, Y.-T. Yang, A 2-D inverse method for simultaneous estimation of the inlet temperature and wall heat flux in a laminar circular duct flow, Numer. Heat Transfer, Part A 34 (1998) 731-745.

[24] F.B. Liu, M.N. Özişik, Simultaneous estimation of fluid thermal conductivity and heat capacity in laminar duct flow, J. Flanklin Inst. 333 (1996) 583-591.

[25] W.K. Cho, D.H. Choi, M.-U. Kim, Optimization of inlet concentration profile for uniform deposition in a cylindrical chemical vapor deposition chamber, Int. J. Heat Mass Transfer 42 (1999) 1141-1146.

[26] W.K. Cho, D.H. Choi, M.-U. Kim, Optimization of the inlet velocity profile for uniform epitaxial growth in a vertical metalorganic chemical vapor deposition reactor, Int. J. Heat Mass Transfer 42 (1999) 4143-4152.

[27] A.K. Alekseev, Freestream parameter estimation using heat flux measurements, AIAA J. 35 (1997) 1238-1240.

[28] A.K. Alekseev, I.M. Navon, The analysis of an ill-posed problem using multi-scale resolution and second-order adjoint techniques, Comput. Meth. Appl. Mech. Eng. 190 (2001) 1937-1953.
[29] F.B. Liu, M.N. Özişik, Inverse analysis of transient turbulent forced convection inside parallel plates, Int. J. Heat Mass Transfer 39 (1996) 2615-2618.

[30] H.-Y. Li, W.-M. Yan, Identification of wall heat flux for turbulent forced convection by inverse analysis, Int. J. Heat Mass Transfer 46 (2003) 1041-1048.

[31] J. Su, A.B. Lopes, A.J. Silva Neto, Estimation of unknown wall heat flux in turbulent circular pipe flow, Int. Commun. Heat Mass Transfer 27 (2000) 945-954.

[32] J. Su, A.J. Silva Neto, Simultaneous estimation of inlet temperature and wall heat flux in turbulent circular pipe flow, Numer. Heat Transfer, Part A 40 (2001) 751-766.

[33] K. Levenberg, A method for the solution of certain nonlinear problems in least-squares, Q. Appl. Math. 2 (1944) 164-168.

[34] D.W. Marquardt, An algorithm for least squares estimation of nonlinear parameters, J. Soc. Ind. Appl. Math. 11 (1963) 431-441.

[35] J.V. Beck, K. Arnold, Parameter Estimation in Engineering and Science, Wiley, New York, 1977.

[36] P. Bradshow, T. Cebeci, J.H. Whitelaw, Engineering Calculation Methods for Turbulent Flow, Academic Press, London, 1981.

[37] M.R. Avelino, J. Su, A.P. Silva Freire, An analytical near wall solution for the $k-\epsilon$ model for transpired boundary layer flows, Int. J. Heat Mass Transfer 42 (1999) 30853096.

[38] D. Coles, The law of the wake in the turbulent boundary layers, J. Fluid Mech. 1 (1956) 191-226.

[39] H. Schlichting, Boundary Layer Theory, seventh ed., McGraw-Hill, New York, 1979.

[40] S.J. Kline, The purpose of uncertainty analysis, J. Fluids Eng. 107 (1985) 153-160.

[41] M.R. Avelino, A.P. Silva Freire, On the displacement in origin for turbulent boundary layers subjected to sudden changes in wall temperature and roughness, Int. J. Heat Mass Transfer 45 (2002) 3143-3153. 